\title{
La comunicación y el enfoque basado en derechos: Una visión desde las Defensorías del Pueblo
}

\author{
María José CAVADAS y María IrÁIzoz LóPEZ-ARRoBA*
}

Propuesto: 12 de marzo de 2012

Evaluado: 30 de marzo de 2012

Aceptado: 18 de abril de 2012

(Abstracts y palabras clave al final del texto)

\section{DESARROLLO Y COMUNICACIÓN}

Los conceptos desarrollo y comunicación llevan conjugándose desde hace muchos años y en su larga andadura han adoptado significados diferentes, según el punto de vista desde el que se los mirase ${ }^{1}$. Sin embargo, cada vez prevalece más la idea de que el desarrollo sería imposible sin el trabajo añadido de la comunicación. Idea que se refuerza si consideramos que el desarrollo es un derecho del ser humano ${ }^{2}$. Perspectiva que se ha impuesto hasta el punto de que la inmensa mayoría de los organismos internacionales cuentan con un componente de comunicación y desarrollo no sólo en materia institucional, sino también en la operativa.

En el presente artículo se ha estudiado la labor de comunicación que realizan las Defensorías del Pueblo en Iberoamérica con el propósito de dar a conocer sus servicios para defender los derechos humanos así como para crear conciencia de cuales son esos derechos. En el curso de la investigación se ha puesto de relieve que el término comunicación utilizado por dichas instituciones no corresponde al proceso real de comunicación. Es decir, no ofrecen un camino de retorno a la opinión del ciudadano. Ni siquiera se han documentado casos concretos sobre comunicación en su sentido más amplio. No obstante, se ha considerado como sinónimo de comunicación el trabajo que realizan en el campo de la educación y de la difusión informativa porque contribuye a crear un clima favorable para desarrollar una conciencia social en materia de derechos humanos y desarrollo. El aprovechamiento de la labor de las Defensorías del Pueblo por parte de los actores de desarrollo es apenas existente, sin que se haya podido documentar.

\footnotetext{
* Universidad Complutense de Madrid

1 Rogers, Everett M. (1976): "Communication and Development The Passing of the Dominant Paradigm", en Communication Research, April vol. 3, 2: 213-240.

2 Resolución de la Asamblea General de Naciones Unidas, 41/128. Declaración sobre el Derecho al Desarrollo, de 4 de diciembre de 1986.
} 


\section{METODOLOGÍA}

La figura del Defensor del Pueblo, pese a tratarse de una institución relativamente joven, goza de gran respaldo social en toda Latinoamérica. Aunque puede que las expectativas despertadas en las clases sociales con menor nivel educativo no queden satisfechas (Carpizo, 1992), la atención que han dedicado a los problemas de los más desfavorecidos les ha otorgado un prestigio del que no gozan ni de lejos otras instituciones gubernamentales.

Debido a esta circunstancia y la penetración en el área latinoamericana, se ha elegido la Defensoría del Pueblo como objeto de estudio de un modelo de institución que utiliza la comunicación como herramienta para promover los derechos humanos. El trabajo de investigación se ha centrado en los mandatos constitucionales de las defensorías y en averiguar hasta qué punto la comunicación está integrada en su quehacer diario.

Para ello se ha utilizado como campo de análisis los portales digitales de dichas instituciones por los siguientes motivos:

1. Ofrecen una completa recopilación de las actividades que realizan en materia de promoción y defensa de derechos.

2. Constituyen un canal accesible para buena parte de la población, especialmente los sectores "ilustrados" que pueden realizar un papel de liderazgo .

3. La penetración de Internet compensa la limitación de oportunidades educativas y culturales que sufre buena parte de la población

4. El lenguaje de Internet se adapta bien a la información de servicio que resulta útil al lector.

5. La estructura de la red facilita que se publiquen artículos comprometidos que difícilmente serían acogidos en los circuitos tradicionales. Ejemplo el artículo publicado en el portal de Guatemala el 8 de febrero de 2012 en el que responsabiliza a agentes del estado de violaciones de derechos.

6. Las generalidad de las Defensorías disponen de un portal digital donde mostrarse a los ciudadanos.

La conciencia de la importancia de la comunicación está tan arraigada que la red de defensorías disponen, como puede comprobarse a través de sus páginas webs, de oficinas de prensa perfectamente articuladas desde las que emiten comunicados de prensa, elaboran boletines, programas radiofónicos y videos cuyos contenidos se refieren en exclusiva a la defensa de los derechos.

\section{ANTECEDENTES}

Aunque, como se ha dicho anteriormente, desarrollo y comunicación caminan de la mano desde hace varias décadas, tanto el concepto de desarrollo como el papel que juega la comunicación en el mismo ha variado. Para empezar y, a modo de resumen, es preciso reconocer que el punto de vista de desarrollo como algo exclusivamente 
relacionado con lo económico y monetario ha ido dando paso a una perspectiva más amplia en la que la protección y promoción de los derechos humanos ha ido ganando terreno. Otro tanto ha ocurrido con la incorporación de la comunicación al proceso.

En los últimos años se han organizado congresos, talleres y seminarios con el objetivo común de tratar el papel de la comunicación en los procesos de desarrollo.

Sin embargo, el hito de mayor impacto en la vinculación entre comunicación y desarrollo fue el primer Congreso Mundial de Comunicación para el Desarrollo, celebrado en Roma en 2006, con la pretensión de demostrar a quienes tienen en sus manos el poder de definir políticas de desarrollo la efectividad de la comunicación para enfrentar los retos más importantes. Por lo tanto, la perspectiva de comunicación debe ser integrada a las políticas y planes. Organizado por la Organización de Naciones Unidas para la Alimentación (FAO), el Banco Mundial y la Iniciativa de la Comunicación, atrajo a más de 900 personas, de las cuales 72 eran expertos en comunicación y cooperación para el desarrollo ${ }^{3}$. En el seminario titulado Sin Comunicación No Hay Desarrollo se demostró la importancia de la co-municación en los avances del desarrollo, identificando cuál es su valor agregado y en qué consiste su aporte e incidencia en los cambios. Los panelistas expusieron una serie muy diversa de estrategias de comunicación, impactos, aprendizajes e interrogantes: estrategias de abogacía y capacitación para incidir en el diseño de políticas públicas de televisión infantil; movilización, monitoreo, análisis y calificación de medios para influir la agenda pública del desarrollo; la recuperación de formatos como la radionovela; la conformación de redes de emisoras que utilizan la radio por satélite; los aprendizajes de la comunicación en salud y los procesos de participación y opinión de la ciudadanía sobre su salud y la de su comunidad; la diferencia entre medios del Estado y medios públicos; el periodismo público para construir agendas ciudadanas, el diálogo entre sociedad civil y el Congreso de la República del Perú para vigilar y colaborar o cogestionar iniciativas y las veedurías de medios, entre otras.

El taller Onda Rural, sobre Radio, Nuevas Tecnologías de Información y Comunicación y Desarrollo Rural, fue otra iniciativa interesante. Organizada por la FAO y la División de Comunicación para el Desarrollo del Banco Mundial junto con otros patrocinadores, se realizó en Quito, Ecuador, entre el 20 y el 22 de abril de 2004. En él participaron 89 expertos en comunicación rural y terminó con la Declaración de los 2800 que definía una guía para colocar las nuevas tecnologías en la agenda de las emisoras radiales, de manera que se cerrase la brecha existente entre el avance de las NTIC y la pobreza rural. También se gestó la publicación de Lo que hay que ver: radio, NTIC e interactividad.

Otras iniciativas en esta misma línea han sido el Taller regional sobre comunicación para el desarrollo y modos de vida sostenibles, celebrado del 28 de mayo al 1 de junio de 2006 en Costa Rica; o el Encuentro internacional de comunicación y desarrollo de los pueblos indígenas, realizado del 13 al 15 de septiembre de 2006, en Santacruz, Bolivia, convocado con el apoyo de la FAO y Calandria, instituciones socias de la Iniciativa de la Comunicación.

3 The Communication Initiative Food and Agriculture Organization of the United Nations (FAO), The World Bank. Lessons, Challenges, and the Way Forward. Roma, 2007. 
Así mismo, es reseñable la publicación realizada entre la FAO y la División de Comunicación del Banco Mundial titulada Teoría y experiencias en comunicación y la administración de recursos naturales. Constituye una herramienta para quienes trabajan o están interesados en este tema, de manera que puedan tener una mejor comprensión de los cambios recientes de las diferentes teorías y estrategias de la práctica actual.

El Seminario celebrado en noviembre de 2006 en Guatemala sobre Habilidades de Comunicación en las Defensorías del Pueblo, en el que participaron los responsables de comunicación de dichas instituciones en Latinoamérica, demostró el interés por renovar los conceptos de comunicación para el desarrollo y la manera de transmitir dichos mensajes. El Seminario supuso, además, el punto de arranque a la colaboración entre las distintas defensorías y una toma de conciencia del nuevo modo de enfocar la comunicación institucional.

La conclusión de estos encuentros es que existe evidencia documentada de los resultados e impactos de abordar los procesos de desarrollo incluyendo estrategias de comunicación y que los principales retos actuales de la humanidad están relacionados con la superación de la pobreza.

El derecho a la comunicación es un derecho humano emergente, que refuerza el derecho a la igualdad y libertad, y está fundamentado sobre el derecho a "investigar y recibir informaciones y opiniones, y el de difundirlas, sin limitación de fronteras, por cualquier medio de expresión", reconocidos en la Declaración Universal de los Derechos Humanos de 1948.

La comunicación para el desarrollo es un proceso social basado en el diálogo que utiliza un amplio espectro de instrumentos y métodos. Busca el cambio a diferentes niveles, como son: escuchar, construir confianza, compartir conocimientos y habilidades, construir políticas, debatir y aprender para lograr un cambio significativo y sostenible. No se trata de relaciones públicas o comunicación corporativa.

Se requiere que las organizaciones responsables del desarrollo le den mayor prioridad a los elementos esenciales de los procesos de la comunicación para el desarrollo, como ha demostrado la investigación y la práctica. Por ejemplo: el derecho y la posibilidad de que la población participe en las decisiones que le afecta; o, asegurarse de que a tiene a su alcance las herramientas de comunicación necesarias para que ella misma se pueda comunicar. Con este objeto cada vez es más concluyente que se debe fortalecer la capacidad de la comunicación para el desarrollo en todos los niveles en el interior de los países y organizaciones. Esto incluye incorporar en las organizaciones especialistas en comunicación para el desarrollo y el personal necesario para el desarrollo de futuros cursos y programas académicos.

Expandir el nivel de inversión financiera para garantizar financiación adecuada y coordinada para los elementos definidos como centrales de la comunicación para el desarrollo, mencionados anteriormente. Esto implica un presupuesto específico para acciones de comunicación, así como implementar políticas y legislación que permita este desarrollo de la comunicación, incluido el uso de medios libres y plurales, según Amparo Cadavid Bringe.

Alfonso Gumucio, consultor internacional y director del Consorcio Comunicación para el Cambio Social afirma que: 
el modelo de comunicación imperante en muchas agencias de cooperación y desarrollo, tanto multilaterales como bilaterales, está caracterizado por la verticalidad y el afán de figuración.

Esta realidad afecta a los proyectos que ejecutan directamente, y también a los que son co-ejecutados con organizaciones e instituciones nacionales y locales. Son muchos los recursos que se invierten en acciones masivas de información que no están articuladas con procesos ni con organizaciones sociales, cuyos resultados son mínimos. En este sentido Gumucio afirma que:

la primera confusión se produce al no establecer distinción entre comunicación e información. Numerosas agencias de desarrollo invierten en programas de información, pero no de comunicación; confunden la difusión a través de los medios masivos con los procesos de comunicación participativa que permiten a las comunidades apropiarse de la comunicación como un derecho.

\section{DESARROLLO INCLUSIVO. LA AGENDA DE EFICACIA DE AYUDA AL DESARROLLO}

El contexto de la ayuda y la cooperación al desarrollo ha cambiado notablemente durante la última década. La denominada Agenda de Eficacia de la Ayuda ha diseñado esta nueva arquitectura internacional de ayuda al desarrollo que se ha ido forjando desde el Consenso de Monterrey sobre financiación del desarrollo (2002), el Foro de Alto Nivel sobre Armonización en Roma (2003) y la Declaración de París sobre eficacia de la ayuda (2005). Se ha consolidado con el Programa de Acción de Accra 2008 y, reafirmado recientemente en Busán, a finales de 2011, en el Cuarto Foro de Alto Nivel sobre la Eficacia de la Ayuda. Este nuevo contexto promueve la idea de una nueva asociación para el desarrollo, inclusiva, basada en principios y propósitos comunes y compromisos diferenciados para un desarrollo internacional efectivo y la premisa de que la promoción de los derechos humanos, la democracia y la gobernabilidad son una parte integral de los esfuerzos del desarrollo 4 .

La representante de ONU-Mujeres en el mismo foro señaló la necesidad de asegurar que las políticas y los programas estén basados en los derechos humanos, con un enfoque en la igualdad de género 5 . En la misma línea, el Foro Global de la Mujeres de Busán declaró que "es crucial ir más allá de la eficacia de la ayuda y hacia una cooperación al desarrollo basada en los derechos humanos como un nuevo marco de solidaridad internacional para avanzar en el desarrollo y la erradicación de la pobreza de

\footnotetext{
$44^{\text {th }}$ High Level Forum on Aid Effectiveness, 29 nov-1 dec 2011, Busan, Korea. Busan partnership for effective development co-operation. Párrafos 1 y 3.

5 Women and Gender Equality in the Aid Effectiveness Agenda. Women and Gender Equality in the Aid Effectiveness Agenda Posted on November 30, 2011. Remarks of Michelle Bachelet Executive Director of UN Women at the Fourth High Level Forum on Aid Effectiveness - Special session on Gender. Busan, South Korea, 30 November 2011.
} 
manera coherente con las normas internacionales de derechos humanos y poniendo debida atención a los derechos de las mujeres, el derecho al desarrollo y la justicia ambiental"6. En paralelo, y de manera complementaria el enfoque basado en derechos humanos tiene cada vez mayor aceptación entre los actores del desarrollo ${ }^{7}$.

Estos avances declarativos en el plano internacional no encuentran expresión en programas concretos. En parte, este desajuste es atribuible no tanto a la falta de voluntad como al desconocimiento de cómo incorporar el enfoque de derechos en estos programas. Es decir, en muchas ocasiones la práctica revela que los actores de desarrollo tienen un conocimiento teórico, pero no disponen de metodologías u orientaciones sobre cómo operativizar estos principios. Y es en este punto donde ayudaría la conexión con las Defensorías del Pueblo porque poseen información cualificada sobre la situación de los derechos humanos.

\section{LA EDUCACIÓN EN DERECHOS COMO MANDATO DEFENSORIAL}

Para defender un derecho vulnerado lo primero que se requiere es tener conocimiento y conciencia de que se posee ese derecho y de que hay un Estado obligado a garantizarlo $^{8}$. En este sentido Valladares ${ }^{9}$ justifica la relevante labor de promoción que han introducido casi todas las Defensorías del Pueblo en América Latina.

La educación y sensibilización en derechos es primordial. Eso incluye también el conocimiento de las garantías que puede ofrecer el Estado y "las obligaciones que implican". Esta labor de promoción de los derechos humanos es trascendental de cara a su protección, en particular en los países del entorno latinoamericano donde los niveles educativos son tan desiguales y existen vastas zonas en las que la presencia del Estado es mínima. En la actualidad esta labor de promoción, no sólo del contenido de los derechos humanos, sino del mandato institucional de las propias Defensorías, ocupan un espacio prioritario en su desempeño. Todas las Defensorías del Pueblo de América Latina tienen este mandato constitucional o legal, a excepción de Argentina, Perú y Puerto Rico quienes lo tienen no como mandato, sino como atribución.

Todas las Defensorías del Pueblo de la región realizan esfuerzos notables para que la ciudadanía conozca y comprenda lo que lo que la institución puede hacer.

6 Declaración política del Foro Global de las Mujeres de Busán, para el 4to Foro de Alto Nivel sobre la Eficacia de la Ayuda (FAN4). Busán, noviembre/diciembre de 2011.

7 Grugel, Jean; Piper, Nicola (2011): “Do Rights Promote Development?” Global Social Policy, vol. 9, no. 1, pp. 79-98. - RED EN DERECHOS. El enfoque basado en Derechos Humanos: nueva mirada a la cooperación: Construcción de ciudadanía y de voluntad política. Documentos para el debate. Diciembre 2010. Acebal Monfort, Luis (coord.). El enfoque basado en Derechos Humanos y las politicas de cooperación internacional. Análisis comparado con especial atención al caso español. Red Enderechos. Madrid.

8 Iráizoz López-Arroba, María (2011): La eficacia de las Defensorías del Pueblo iberoamericanas en la protección de los derechos humanos: Estudio comparado. Tesis doctoral. Departamento de Fundamentos del Derecho y Derecho Penal. Facultad de Derecho. Universidad de Alcalá. Marzo . p. 237.

9 Valladares Lanza, Leo. "The challenges facing the Ombudsman in Latin America". En: The International Ombudsman Yearbook, vol.2, International Ombudsman Institute and Linda C. Reif, The Hague: Kluwer Law International, 1998, p. 159-165. 
Esto es lógico ya que son instituciones jóvenes, que rondan en su mayoría los veinte años. Cuanta más información exista sobre las Defensorías y sus mandatos, mayor será la eficacia de éstas. Como afirma Carpizo ${ }^{10}$, quizá la más importante función del Ombudsman sea la de educar.

\section{LA LABOR DE LAS DEFENSORÍAS DEL PUEBLO (TAMBIÉN DENOMINADAS INSTITUCIONES NACIONALES DE PROMOCIÓN Y PROTECCIÓN DE LOS DERECHOS HUMANOS INDH)}

La mayor parte de las Defensorías del Pueblo nacen a principios de los años 90, salvo las de Portugal, España y Guatemala que se crearon constitucionalmente en 1976, 1978 y 1985, respectivamente. Tienen por mandato la protección de los derechos fundamentales y en el caso de las Defensorías de América Latina tienen, en su inmensa mayoría, el mandato expreso de contribuir a la defensa y promoción de los derechos humanos y soportan esta labor desde los pilares de la educación y de la comunicación.

En el caso de Guatemala, por poner un ejemplo, que se hace extensivo al resto del área, se declara que el Procurador tiene, entre otras, las siguientes atribuciones:

1.- Promover y coordinar con las dependencias responsables para que en los programas de estudio de la educación oficial y privada se incluya la materia específica de los Derechos Humanos que deberá ser impartida en horarios regulares en todos los niveles educativos.

2.- Desarrollar un programa permanente de actividades para que examinen aspectos fundamentales de los derechos humanos y se realicen informes, compilaciones, estudios, investigaciones jurídico-doctrinales, publicaciones, campañas divulgativas y cualquier otra actividad de promoción, con el propósito de hacer coincidir en los diversos sectores de la población sobre la importancia de estos derechos.

3.- Establecer y mantener la comunicación con las diferentes organizaciones intergubernamentales y no gubernamentales, nacionales o extranjeras, encargadas de la defensa y promoción de los derechos humanos.

Las Defensorías del Pueblo en Latinoamérica han asumido el papel educativo que les corresponde en la difusión de los derechos humanos. Para ello destinan gran parte de sus recursos a la edición de material informativo y realizar programas de difusión. Aunque en la mayoría de los casos se trata de un proceso informativo, en tanto que la información circula en un único sentido, desde las Defensorías a los destinatarios, sin ofrecer la posibilidad de que éstos puedan hacer retornar sus opiniones, se trata de un material informativo reseñable que está creando una base bibliográfica documental de referencia que, a su vez, contribuye a expandir un clima de reflexión sobre derechos humanos y desarrollo.

10 Carpizo, Jorge (1998): Derechos humanos y Ombudsman. 2. a edición, México: Ed. Porrúa y UNAM, 1998. página 58. 
Los portales oficiales de las Defensorías del Pueblo constituyen el mejor exponente de esta labor educativa e informativa. Las tres cuartas partes de los contenidos lo componen una serie de documentos que recogen la actividad concreta que realizan sobre el terreno. Entre el material albergado en las webs, destacan los informes defensoriales que cada año presentan al Parlamento nacional correspondiente. Constituyen un inventario de actividades encaminadas a poner de relieve algunos de los problemas de derechos humanos más acuciantes. Estos informes incorporan recomendaciones a los gobiernos para que dispongan las soluciones más adecuadas que contribuyan a mejorar el nivel de disfrute de los derechos.

Por otra parte, cabe subrayar la labor de las oficinas de prensa para reforzar la tarea de difusión. Forma parte de su naturaleza transferir a los medios de comunicación tradicionales el trabajo que realiza la Defensoría en materia de derechos humanos, pero los comunicados y notas de prensa, constituyen en sí mismos un trabajo paralelo en la misión de destacar los derechos de la población: derechos a la educación, la salud, una vivienda, entre otros.

En algunas defensorías, las oficinas de prensa realizan un trabajo añadido al mandato constitucional y han diseñado programas radiofónicos o editan vídeos cuyos contenidos se refieren a casos concretos de violación de los derechos. Argentina es la Defensoría prototipo en la gestión de la comunicación en la doble vertiente; la dirigida a los gobernantes $\mathrm{y}$, por otra parte, a los ciudadanos.

Además de lo reseñado anteriormente, las Defensorías editan trípticos y folletos informativos generalmente sobre asuntos de salud y de educación, además de publicaciones más amplias sobre la defensa de los derechos.

Aunque el diseño de las páginas posiblemente no obtendrían premio en un certamen de páginas web, se percibe que las Defensorías han hecho un esfuerzo por imponer un sentido práctico al espacio y que resulte fácil acceder a la información.

Sin embargo, se echa en falta en estos países, donde todavía existen barreras en el acceso a la educación, sobretodo en términos de calidad y equidad y, donde a pesar del crecimiento de la telefonía móvil, no existe un desarrollo económico que permita la aplicación de la tecnología digital, la adopción de métodos educativos que puedan llegar más fácilmente a la población.

Este decálogo se hace extensivo al resto de las Defensorías estudiadas. Si bien la forma de aplicarlo varía según cuales son los problemas más comunes en cada país.

\section{PANAMÁ}

La home de la web de la Defensoría de Panamá, se ordena según el tipo de publicaciones y comunicados que emite. Una pestaña remite a la colección de notas orientativas, otra a los artículos penitenciarios, la tercera a la colección de leyes y decretos, la cuarta pestaña a la colección de revistas penitenciarias y una quinta a libros y manuales penitenciarios.

Las noticias remitidas a la prensa tienen un espectro de temas más amplio que los asuntos carcelarios, pero el común denominador, son los derechos humanos. Titulares como "Casos de pinchazos telefónicos preocupa a la Defensoría", "Inspeccionan el vertedero municipal de Cerro Patacón para detectar a menores de 
edad que laboran", "Falta de antirretrovirales atenta contra los derechos humanos y la vida de las personas con VIH/Sida", "Oficinas regionales realizan inspecciones para detectar trabajo infantil" son habituales en la página oficial.

La pestaña de Publicaciones agrupa leyes, informes, boletines, constitución y trípticos. En cada uno de estos apartados la temática es de la misma naturaleza, si bien varía el tipo de documento, extensión y a quien va dirigido. Por ejemplo, el informe especial del Defensor sobre elusión en el pago de pensiones alimenticias y mecanismos de garantía del derecho de alimentos, datado en Ciudad de Panamá en noviembre de 2009, puede encontrarse junto a un informe preliminar sobre los incidentes en la provincia de Bocas Toro en julio de 2010 en el que la Defensoría realiza un análisis de los incidentes ocurridos ante la reclamación de obreros afiliados al sindicato de trabajadores de la industria del banano de la empresa Bocas Fruitcompany en la provincia de Bocas del Toro.

En la biblioteca virtual se encontrarán publicaciones con los siguientes títulos: Violencia doméstica, Todos tenemos derechos, Prevengamos el VIH-SIDA, Policía y Derechos Humanos, Personas con Discapacidad Derechos y Oportunidades. Asimismo incluyen vídeos explicativos sobre cuestiones de orden práctico como qué es la Defensoría del Pueblo, Cómo se presenta una queja o quienes puede presentar una queja en la Defensoría.

\section{ECUADOR}

En el caso de Ecuador, las noticias tienen un carácter más personalista. Generalmente resaltar el protagonismo de los defensores o de los consejeros. Por ejemplo, se encuentran reseñas de la reunión de la presidenta de la Defensoría con el Comité Directivo, la Defensoría promueve la conformación de las asambleas ciudadanas, el concurso público de oposición para designar defensor del Pueblo. Asimismo se otorga especial relevancia a los Observatorios Ciudadanos.

Un contenido común en la mayoría de las Defensorías es la presencia de una pestaña dedicada a la ley de transparencia donde se explica el estado de cuentas y la contratación de personal.

Los boletines de prensa informan de la situación de Veedor Internacional Baltasar Garzón, recogen el listado de catedráticos que elaborarán un banco de preguntas para los concursos de la Contraloría, Defensoría Pública y Tribunal Contencioso Electoral, o de la rendición de cuentas ante la ciudadanía.

\section{GUATEMALA}

Guatemala continúa el mismo tono institucional, con informaciones como la celebración del Día Internacional de la Lengua Materna, proclamado por la Organización de las Naciones Unidas para la Educación, la Ciencia y la Cultura (UNESCO) en noviembre de 1999, o el informe anual de la Defensoría ante el Congreso de la República.

Frente al oficialismo de los informes, resulta curioso destacar la radionovela "Carolina, el largo camino de la dignidad" entre otros. Además de los resúmenes ejecutivos más relevantes sobre Derecho a la Educación, a la Alimentación o las de- 
sapariciones forzadas. La Defensoría emite informes ejecutivos sobre casos paradigmáticos. Entre los últimos remitidos se encuentran el Caso Químicos, el Aumento de la canasta básica de alimentos o del hospital San Juan de Dios. En cada uno de ellos destacan la posición de debilidad de la población.

En un artículo fechado el 8 de febrero de 2012 se reconoce que las violaciones de derechos humanos son cometidas por autoridades de agentes del Estado, grupos de poder político y económico o personas particulares con el fin de mantener privilegios y control de situaciones que producen beneficios para unos e injusticias y sufrimientos para muchos.

El caso de una mujer maltratada llama la atención por su fuerza y estilo narrativo en primera persona. "Anoche me volvió a golpear y me amenazó con matarme. Ni el maquillaje ni las mangas largas podrán esconder los cortes y golpes que me ocasionó esta vez". Es decir, además de la difusión de contenidos informativos sobre los derechos humanos, las defensorías sirven de plataforma para dar a conocer experiencias de la población víctima de la violencia.

\section{COSTA RICA}

La web de Costa Rica muestra sus credenciales en la home. Lo primero que encuentra el visitante es la pestaña de acceso a la red Interinstitucional de Transparencia, a continuación la pestaña del Mecanismo Nacional de Prevención de la Tortura y el Foro permanente sobre Población Migrante y Refugiada. Así mismo dispone de un link titulado "Tú puedes parar la corrupción 2011 Año Internacional de las personas Afrodescendientes".

La mayoría de los contenidos, ya sean el calendario de actividades, de actualidad informativa, boletines de prensa o los informes anuales de actividades, mantienen el objetivo común de proteger y defender los derechos humanos. "Condena a hacienda por violentar los derechos de habitantes y trabajadores", "Informe final de las Torres de Telefonía Celular", "Pronunciamiento de la Defensoría ante la realización de pruebas de bachillerato", son algunos de los títulos.

El papel de la Defensoría como contrapunto al poder gubernamental queda de manifiesto en los Boletines de prensa: "Defensoría cuestiona proceso para alzas en servicio de agua", "Defensoría se pronunciará ante el paro del Sector Público", "Defensoría pide al Gobierno apoyo a proyectos de ley de unión civil", son algunos de los que pueden encontrarse.

Ningún aspecto de la actualidad afectado por un conflicto social queda fuera del área de actuación de la Defensoría del Pueblo que investiga cada asunto y realiza un diagnóstico de la situación. Cada uno de estos casos da lugar a informes que por sí mismos constituyen un fondo documental y contribuyen a la difusión de los derechos humanos.

\section{PERÚ}

Ofrece una web muy práctica para moverse. En primer lugar anuncia el Centro de Información con una línea de teléfono gratuita y el modo de presentar una queja. 
Una explicación detallada de cuales son los casos en los que puede intervenir la Defensoría: casos relacionados con la libertad personal, la seguridad ciudadana, transparencia y acceso a la información pública, prevención de la corrupción, recomendaciones para contribuir a mejorar el sistema de justicia y quejas de vecinos, castigo físico, violencia sexual a niños y adolescentes, tratamiento a los pueblos indígenas, a personas privadas de libertad, así como seguimiento de la situación de los peruanos en el exterior. Cada uno de los asuntos que pasan por la Defensoría se convierte en materia susceptible de convertirse en noticia: es decir, salta a los medios de comunicación, da lugar a un informe y entra a formar parte de los Informes Defensoriales. En definitiva, constituye un reguero informativo que ahonda en ese clima social de defensa de los derechos.

\section{MÉJICO}

La web de la Defensoría del Pueblo de Méjico es paradigmática en disponer los contenidos como un universo informativo perfectamente organizado. Los documentos emitidos bien en forma de cartillas de derechos bien en forma de guías prácticas, los informes especiales sobre Seguridad e Higiene en una cuenca carbonífera o sobre el secuestro de emigrantes define la vocación educativa y de difusión. Por si quedara alguna duda de ello se permite editar una guía para implementar medidas cautelares en beneficio de periodistas y comunicadores en Méjico.

Anteriormente nos hemos referido al diseño de las webs de las Defensorías como un factor secundario para sus responsables, más interesados por la utilidad que por la estética. El portal mejicano es un ejemplo paradigmático. En un solo clic, el visitante tiene a la vista los elementos más solicitados: información y mecanismos de consulta. La pestaña de sobre cómo presentar una queja es un ejemplo de información de servicio. La Defensoría anuncia hasta donde llega su competencia para tramitar una queja, al tiempo que se declara competente cuando las autoridades administrativas de carácter federal, con excepción del Poder Judicial de la Federación, cometan actos u omisiones que violen los derechos humanos. Asimismo aporta números de teléfonos y de faxes a los que dirigir reclamaciones los 365 días del año las 24 horas del día. Las gacetas, publicadas desde 1990, y que pueden encontrarse en la web, constituyen informes detallados de la actividad de la institución.

\section{ARGENTINA}

La web de la Defensoría de Argentina destaca por su potente sentido mediático. La mayor parte de los contenidos son presentados de forma que atraigan la atención del visitante. Las actuaciones del Defensor del Pueblo se presentan con un gran despliegue fotográfico de manera que a veces parece que se está ante un periódico digital. Las noticias de actualidad, bien apoyadas en soporte gráfico, se suceden unas a otras, de manera que el visitante tiene la sensación de estar ante una home permanentemente refrescada. Es un ejemplo de cómo un portal que en principio, por el tipo de institución, podría pecar de estático se transforma en algo dinámico, más propio de un medio de comunicación digital. 
Contribuye a esta sensación el hecho de que la oficina de prensa inserta una galería de fotos, video, spot para televisión y hasta un programa de radio con pod cast que pueden descargarse. El programa comenzó a emitirse en 1999 y no ha sufrido interrupciones. "La gente y su defensor", es difundido de manera gratuita y se transmite por más de 200 emisoras en todo el país, desde Jujuy a Tierra de Fuego.

\section{ESPAÑA}

El portal español recoge en su home tres elementos básicos: un número de teléfono al que acudir, un link para presentar la queja online, una relación de los derechos del ciudadano y el acceso a los servicios de prensa y publicaciones. Por supuesto, como se repite en las anteriores webs, un espacio de actualidad con las noticias más sobresalientes están relacionadas con la actividad de la institución y de la Defensora.

Un centro de documentación y publicaciones bien organizado, pone a disposición del interesado los informes anuales, mediante los cuales el Defensor del Pueblo tiene que dar cuenta a la Cortes Generales de la gestión realizada. Además, los informes monográficos que estudian problemas de la sociedad española en relación con los derechos y deberes proclamados por la Constitución.

Las recomendaciones de la Defensoría se realizan cuando una norma o ley tiene consecuencias injustas para las personas. Las recomendaciones se pueden encontrar en la pestaña de publicaciones. Igualmente se encuentran allí el régimen jurídico que soporta al Defensor, así como otras publicaciones editadas por el Defensor o en colaboración con otras instituciones y que recogen la participación en congresos y jornadas.

\section{BOLIVIA}

La web de Bolivia recibe al visitante con una poderosa llamada de sensibilización en forma de cuña parpadeante y dinámica, incluso con papel preponderante al logo institucional: "Todas las personas tenemos los mismos derechos". "Por la defensa de la vida y la dignidad de las personas".

Las noticias de actualidad de la propia Defensoría (actividades del Defensor, cambio de horarios, nombramientos, jornadas) publicaciones e informes recientes de la institución reciben protagonismo.

La web acusa la influencia de la primera Defensora del Pueblo de Bolivia, Ana María Romero del Campero, reputada comunicadora social que desde principio imprimió una clara vocación comunicadora a la institución. Es una página ordenada donde las pestañas tienen buena identificación y visibilidad. Destaca la campaña: Ninguna persona es ilegal, que busca promover un mensaje de unidad dentro del cual los derechos humanos son iguales para todos, más allá del origen o condición de cada persona y del lugar donde se encuentre. Entre los materiales producidos hay una selección de trípticos, folletos y afiches producidos por la Defensorías desde el año 2006. En total se exponen más de 50 producciones. Además de su página web, la Defensoría tiene una extensa actividad de comunicación social en radio, prensa y televisión. Algunas cuñas y vídeos se ven desde el portal. 


\section{COLOMBIA}

El portal de la Defensoría del Pueblo de Colombia es uno de los más activos y actualizados de la región donde la mayor parte de la información está a la vista. Posiblemente peque de demasiada densidad en la página principal, lo que resta visibilidad a otros aspectos importantes. En su parte central se ubican todas las noticias referidas a derechos humanos y derecho internacional humanitario y su vulneración; todas ellas están vinculadas al conflicto armado.

Contiene una rica y variada oferta de materiales de formación en derechos humanos: Acciones populares y de grupo, Agua, Derecho a la vida, Derecho al Trabajo, Derecho Internacional Humanitario, Derechos Humanos, Desaparición Forzada, Desplazados, Discriminación, Educación, Grupos en condiciones de vulnerabilidad, Informes al Congreso, Justicia, Medio Ambiente, Mujer, Personas privadas de la libertad, Salud, Sistema de Alertas Tempranas, Sistema de Seguridad Social, Violencia intrafamiliar. Especialmente relevantes para el desarrollo son las cuartillas elaboradas dentro del Programa de Seguimiento y Evaluación de las Políticas Públicas en Derechos Humanos que realiza la Defensoría del Pueblo, (PROSEDHER). Algunos ejemplos de éstas son: Informe del derecho humano a la educación; Las Politicas Públicas Alimentarias en Colombia. Un Análisis desde los Derechos Humanos; El derecho humano al agua; o el Protocolo para incidir en la gestión del seguimiento y evaluación de la política pública con enfoque de derechos en lo regional y local. Todas las publicaciones y producciones elaboradas en el marco de este programa están vinculadas al ejercicio de los derechos económicos, sociales y culturales y por tanto, tienen especial relevancia en el desarrollo humano.

\section{EL SALVADOR}

El portal de la Procuraduría de Derechos Humanos de El Salvador adopta un tono institucional y sobrio. No se reconocen elementos de promoción de derechos humanos. Su contenido hace referencia a información institucional, la labor y servicios. Otro elemento es la información ordenada por las Procuradurías adjuntas temáticas (niñez y juventud; mujer y familia, medio ambiente, derechos civiles e individuales y derechos económicos, sociales y culturales). Relaciones externas, memoria histórica, centro de prensa y pronunciamientos y publicaciones son el resto de contenidos en el eje horizontal de la web. Los contenidos de esta última son en su mayoría comunicados, informes, estudios o pronunciamientos específicos del Procurador. El informe anual de labores que se presenta a la Asamblea Legislativa. Los informes especiales emitidos son muy variados, abarcan desde la situación de la mujer privada de libertad, o el caso de los jesuitas asesinados en 1989, como la desaparición forzada, o el Informe sobre el estado del derecho a la alimentación en El Salvador elaborado con el apoyo de la Fundación Ayuda en Acción.

\section{NICARAGUA}

La Procuraduría de Derechos Humanos de Nicaragua presenta un portal muy sencillo con un contenido esencialmente institucional: misión y valores, cómo pre- 
sentar una queja; qué derechos se tutelan y una sección dedicada a los informes anuales emitidos. Tiene una sección de noticias muy obsoleta, la mayoría corresponden al año 2008. La institución de Nicaragua es probablemente la más débil institucionalmente del entorno iberoamericano y esta situación se refleja claramente en su página web.

\section{HONDURAS}

La web del Comisionado Nacional de los Derechos Humanos (CONADEH), está enfocada sobre las noticias de actualidad o recientes pronunciamientos en el centro de su página.

Es una pagina que indica rápidamente al lector cómo poner una denuncia en línea o telefónica. La visibilidad del número gratuito y el enlace de llamada que figura a la izquierda destaca con respecto al resto del contenido. Su estructura es muy sencilla y visual: Inicio; Información; Noticias; Programas Especiales; Quejas y Denuncias. Ofrece una imagen limpia y aporta claridad. La mayoría de las noticias corresponden a pronunciamientos o posicionamientos del Comisionado con relación a temas de actualidad. Es un portal centrado en la difusión de la institución y las actuaciones de su titular. Las noticias de interior no han sido actualizadas desde 2010. La web apenas contiene elementos comunicativos de promoción de derechos humanos.

\section{PARAGUAY}

La primera impresión del usuario que se acerca a la web de la Defensoría del Pueblo de Paraguay es la proximidad de la persona del Defensor del Pueblo, quien aparece en fotografías dinámicas en actitudes cercanas a las personas que le rodean pero profesionales. Logran transmitir la imagen de que lo importante es la labor. Las tres funciones de la institución se indican de manera clara y visible junto a las imágenes. Se mantiene la nitidez en la estructura del portal. La sección de Inicio cuenta con un cuadro central de noticias de actualidad referida a la actividad de la propia Defensoría y de otros organismos afines, también internacionales. Sin embargo, la recepción de quejas recibe la mayor visibilidad y atención.

Al margen del mandato institucional y las actuaciones de la Defensoría, no hay ningún mensaje de promoción de derechos humanos y las noticias han quedado viejas. La página tiene un estilo muy administrativo.

\section{VENEZUELA}

El portal de Venezuela es dinámico y visual. Se articula en torno a dos barras. La horizontal con las pestañas de: Institucional; Publicaciones; Comunicaciones; Denuncias; Programas; preguntas frecuentes y contrataciones. La vertical con acceso a las pestañas Jurídico; en acción; Multimedia; Educando en DDHH y enlaces de interés.

Los contenidos más relevantes en términos de comunicación son: la revista mensual "Lápiz de Pueblo", de difusión institucional; una serie sobre Derechos 
Humanos, unos Manuales de facilitación de derechos humanos; y unos títulos referidos a la Memoria Histórica. Se aporta profusa información sobre las acciones de formación e investigación de la Fundación Juan Vives Suriá, incluidos cursos específicos de 20 horas de duración sobre derechos de la mujer, de pueblos indígenas, o de niños, niñas y adolescentes, entre otros. Además de incorporar una importante oferta educativa y abundante información institucional, cuenta con mensajes claros de promoción de derechos humanos o de incidencia en desarrollo. Interesantes son los enlaces a los programas de radio de "la voz de la Defensoría en la radio", con la lista de diales y horarios de programación nacional o regional y los informes anuales desde 2001.

\section{PUERTO RICO}

El portal de la Oficina del Procurador del Ciudadano de Puerto Rico capta al visitante con las tres prioridades temáticas de la institución: Asuntos ambientales; asuntos hídricos; y Pequeños negocios. La información es sobre todo institucional, explicativa de las distintas instancias que la integran y dirigida hacia cómo realizar una reclamación.

\section{PORTUGAL}

El portal del Proveedor de Justicia de Portugal es sobrio y claro en su información. Esta Defensoría no tiene un mandato de promoción y difusión de derechos humanos y, consecuentemente, no se incluye mensajes o contenido en ese sentido. Sin embargo, sigue una estructura ordenada, con información sobre la institución, su origen y expansión, su funcionamiento y mandato. Destaca sin embargo, su labor en dos líneas especiales, de infancia y personas mayores. Con referencia a ambas publica todos los recursos, con ejemplos y casos prácticos de cómo puede intervenir la institución.

\section{PROPUESTAS: LA COMUNICACIÓN COMO HERRAMIENTA DE CAMBIO}

De todo lo expuesto se concluye que las Defensorías del Pueblo constituyen un instrumento muy adecuado para cumplir la misión amparada por los organismos internacionales de promover y proteger los derechos humanos de las poblaciones de sus países respectivos. Sin embargo, por su reciente creación, o por su fragilidad según los casos, es una institución desconocida para una parte importante de los actores de desarrollo.

Para ello deberían incorporar la comunicación como herramienta capaz de crear conciencia de que existen derechos propios del ser humano que deben reconocerse y reclamarse como algo consustancial a la condición y dignidad humana. Este trabajo sostiene que un mayor acercamiento entre los actores de desarrollo (ONGD, agencias internacionales y bilaterales) y las Defensorías del Pueblo, mediante inter- 
cambio de informaciones, valoraciones, perspectivas e incluso con una participación recíproca en los programas e intervenciones de cada uno, contribuiría a una mayor eficacia en la ayuda al desarrollo de los pueblos.

Uno de los ejemplos más claros sobre cómo optimizar el acervo de derechos humanos desde las Defensorías del Pueblo por parte de los actores de la cooperación y desarrollo lo evidencia la utilidad del "Programa de Seguimiento y Evaluación de las Políticas Públicas en Derechos Humanos, ProSeDHer", elaborado por la Defensoría del Pueblo de Colombia. Busca evaluar las políticas públicas desde una perspectiva de derechos humanos que integre los derechos económicos, sociales y culturales. $\mathrm{La}$ información que recoge este Programa sin lugar a dudas es de extraordinario valor para las ONGD que actualmente trabajan en el país y que seguramente muchas desconocen por la todavía limitada imbricación práctica entre los operadores de derechos humanos y los de desarrollo.

En términos de comunicación, las Defensorías no han logrado ver en ella una herramienta que puede cumplir la labor transformadora hacia un auténtico cambio social. Quizás por desconocimiento, la comunicación aparece en los programas y actividades como sinónimo de información, difusión, promoción o educación y ni siquiera las Defensorías más proclives a incorporar fórmulas informativas proactivas y novedosas la aplican en su acepción más completa. A la vista del estado de la cuestión se propone:

1. Diseñar productos de comunicación acomodados a las necesidades locales, inspirados en la mentalidad y hábitos de los ciudadanos de cada región.

2. Fomentar el uso de los canales más accesibles a los ciudadanos, independientemente de que se utilicen otros canales más restrictivos.

3. Propiciar modelos de información interactivos para que los ciudadanos contribuyan con sus aportaciones a moldear el diseño.

4. Acomodar la actividad comunicativa a la agenda de los ciudadanos, de manera que la incorporen a sus rutinas de vida.

5. Crear equipos de escucha activa capaz de recoger el sentir de los pueblos, con el fin de agregar este conocimiento a los programas de comunicación.

6. Propiciar un clima de confianza a través de oficinas de información, valoración o foros de debate donde los ciudadanos se sientan reconocidos y valorados.

7. Para todo ello se necesita incorporar a las Defensorías auténticos comunicadores, capaces de transformar todo el conocimiento recogido en la "escucha activa" en programas de promoción y defensa de derechos. la labor de comunicación se complementará con otra de seguimiento de los programas.

8. Por último y no menos importante, se recomienda que la cooperación internacional ayude al fortalecimiento en habilidades de comunicación Se trataría de un acercamiento desde los actores del desarrollo a los productos de comunicación, difusión e información, elaborados por las Defensorías, y facilitar su distribución en formatos adecuados a las necesidades culturales y posibilidades económico/tecnológicas de la población destinataria. Supondría aportar un valor agregado en la incorporación del enfoque basado en derechos humanos en las intervenciones de desarrollo. 


\section{CONCLUSIONES}

Del estudio de las páginas webs de las defensorías del Pueblo, se extraen las siguientes conclusiones:

1. Las Defensorías del Pueblo de Iberoamérica parten de una posición de privilegio respecto a cualquier otra institución pública o privada, para desarrollar tareas de promoción de derechos.

2. Las Defensorías, como instituciones nacionales de derechos humanos generan y tienen acceso a información de primera mano sobre las condiciones y contextos de vulnerabilidad que más aquejan a la ciudadanía. Disponen del marco normativo necesario para la clasificación de derechos vulnerados.

3. Todas ellas realizan una gran labor pedagógica y de difusión sobre derechos y la posibilidad de reclamarlos. Se observan, sin embargo, importantes carencias tanto en la pedagogía empleada como en los canales de difusión.

4. Pese a reconocer el valor estratégico de la comunicación en la defensa de los derechos humanos, las Defensorías no han articulado una estructura de comunicación como tal y se limitan a una mera labor informativa.

5. La mayor parte de las publicaciones editadas se refieren a la emisión de informes y publicaciones en los que recogen el inventario de actividades y análisis que realizan, lo cual crea una base documental de referencia porque son instrumentos rigurosos.

6. Apenas se observa articulación entre los actores de desarrollo y la labor de comunicación de las Defensorías. Un mayor aprovechamiento de los primeros de los análisis e informes de las Defensorías optimizaría el trabajo global hacia el desarrollo humano.

\section{REFERENCIAS BIBLIOGRÁFICAS}

\begin{tabular}{|l|l|}
\hline http://www.dhr.go.cr & http://www.argentina.gob.ar/directorio/116-defensor \\
http://www.defensordelpueblo.es & http://www.defensoria.gob.bo \\
http://www.cndh.org.mx & www.procuraduriaddhh.gob.ni \\
http://www.defensoria.gob.pe & http://www.defensoriadelpueblo.gov.py \\
http://www.participacionycontrolsocial.gob.ec/web & http://www.dpn.gob.ar \\
/guest/defensor-del-pueblo & www.conadeh.hn \\
http://www.pdh.org.gt & $h \mathrm{http://www.defensoria.gob.ve}$ \\
http://www.ombudsmanpr.com & http://www.provedor-jus.pt \\
\hline
\end{tabular}

4th High Level Forum on Aid Effectiveness, 29 nov-1 dec 2011, Busan, Korea.

Busan partnership for effective development co-operation. Párrafos 1 y 3.

Acebal Monfort, Luis (coord.).(2011): El enfoque basado en Derechos Humanos y las políticas de cooperación internacional. Análisis comparado con especial atención al caso español. Red Enderechos. Madrid.

Cadavige Bringe, Amparo (2006): Cual Desarrollo. Comunicación para el Congreso Mundial de Comunicación para el Desarrollo. Colombia. 
CARPIZO, Jorge (1992): Algunas reflexiones sobre el ombudsman y los Derechos Humanos. México, D. F.:Dirección de Publicaciones de la Comisión Nacional de Derechos Humanos.

Declaración Universal de los Derechos Humanos de 1948.

Grugel, Jean; PIPER, Nicola. "Do Rights Promote Development?" Global Social Policy, vol. 9, 1, pp. 79-98.

FORESTI, Marta, ET AL (2006): Aid effectiveness and human rights: strengthening the implementation of the Paris Declaration. Overseas Development Institute. Londres, .

Gumucio, Alfonso y Rodriguez. Clemencia (2006): Time to call things by their name: the field of communications. Ed. Social Change.

Resolución de la Asamblea General de Naciones Unidas, 41/128. Declaración sobre el Derecho al Desarrollo, de 4 de diciembre de 1986.

Declaración política del Foro Global de las Mujeres de Busán, para el 4to Foro de Alto Nivel sobre la Eficacia de la Ayuda (FAN4). Busán, noviembre/diciembre de 2011.

IrÁizoz LóPEZ-ARrobA, María (2011): La eficacia de las Defensorías del Pueblo iberoamericanas en la protección de los derechos humanos: Estudio comparado. Tesis doctoral. Departamento de Fundamentos del Derecho y Derecho Penal. Facultad de Derecho. Universidad de Alcalá. Marzo.X.

Red en Derechos (2010): El enfoque basado en Derechos Humanos: nueva mirada a la cooperación: Construcción de ciudadanía y de voluntad política. Documentos para el debate. Diciembre.

Lessons, Challenges, and the Way Forward. The Communication Initiative Food and Agriculture Organization of the United Nations (FAO), The World Bank. Roma, 2007.

Rogers, Everett M (1976): "Communication and Development The Passing of the Dominant Paradigm”, en Communication Research, April, vol. 3, 2: 213-240.

VALLADARES LANZA, Leo (1976): "The challenges facing the Ombudsman in Latin America". En: The International Ombudsman Yearbook, vol.2, International Ombudsman Institute and Linda C. Reif, The Hague: Kluwer Law International.X.

Women and Gender Equality in the Aid Effectiveness Agenda.(2011): Women and Gender Equality in the Aid Effectiveness Agenda Posted on November 30. Remarks of Michelle Bachelet Executive Director of UN Women at the Fourth High Level Forum on Aid Effectiveness - Special session on Gender. Busan, South Korea, 30 November. 


\title{
RESUMEN
}

Reducir la de pobreza, el subdesarrollo y eliminar el analfabetismo en el que viven grandes masas de la población requiere de un enfoque de desarrollo más allá del que se ha aplicado hasta ahora. Las actuaciones dirigidas a atender las necesidades básicas de los desfavorecidos se han demostrado más que insuficientes. Se impone un enfoque nuevo basado en el reconocimiento de los derechos humanos de las personas. Bajo esta perspectiva, que puede significar un cambio de paradigma, la comunicación es un elemento estratégico. Un nuevo modelo de gobernanza basado en la transparencia y la responsabilidad social exige la implantación de redes de comunicación que actúen en el espacio intermedio situado entre los titulares de los derechos y titulares de obligaciones y responsabilidades. Las Defensorías del Pueblo, instituciones con fuerte implantación en la comunidad hispanohablante, pueden cumplir un papel clave en este propósito. Además de tratarse de entidades de rango constitucional y de fuerte prestigio social, cuentan con experiencia en la comunicación para el desarrollo. El presente artículo investiga el trabajo realizado en este sentido y plantea la posibilidad de convertirlos en agentes del cambio para estimular una nueva mentalidad.

Palabras clave: derechos sociales, promoción, Defensorías del Pueblo, comunicación, desarrollo, transparencia.

\begin{abstract}
Reducing poverty, underdevelopment and the eradication of illiteracy among part of the world population requires further approach to development which has been applied so far. The actions aimed at meeting the basic needs of the disadvantaged but have proven insufficient. It imposes a new approach based on the recognition of human rights. From this perspective, that may mean a change of paradigm, communication is a strategic element. A new model of governance based on transparency and social responsibility requires the implementation of communication networks operating in the intermediate space located between holders of rights and holders of obligations and responsibilities. The Defensorias del Pueblo (Ombudsman), institutions with strong presence in the Hispanic community can play a key role in this endeavor. In addition to being constitutional institutions and having strong social prestige, have experience in communication for development. This article investigates the work done in this regard and raises the possibility of turning them into agents of change to stimulate the new paradigm.
\end{abstract}

Key words: social rights, promotion, Ombudsman, communication, development, transparency.

\section{RÉSUMÉ}

Réduire la pauvreté, le sous-développement et 'éliminer l'analphabétisme dans les grandes masses de la population vivant exige une approche de développement au-delà de celle appliquée jusqu'ici. Les actions réalisées auprès des personnes défavorisées se sont montrées les plus inadéquates.On applique ici une nouvelle approche basée sur la reconnaissance des droits fondamentaux des personnes. Dans cette perspective, que peut signifier un changement de paradigme, la communication est un élément stratégique. Un nouveau modèle de gouvernance basé sur la transparence et la responsabilité sociale des entreprises précise la mise en œuvre de réseaux de communication dans l'espace intermédiaire situé entre les détenteurs des droits et des devoirs et des responsabilités. Les médiateurs du peuple, avec une forte présence dans la communauté de langue espagnole, et les institutions peuvent jouer un rôle clé dans ce but. En plus d'être des entités constitutionnelles avec prestige social fort et rang, ils ont l'expérience en communication pour le développement. Cet article examine le travail accompli à cet égard et soulève la possibilité de les transformer en agents de changement afin de stimuler une nouvelle mentalité

Mots clé: droits sociaux, defenseur du peuple, promotion, communication, developpement, stretégies. 